ARTICLE

\title{
Tetrasubstituted imidazoles as incognito Toll-like receptor 8 a(nta)gonists
}

Yi Yang (1) 1,5, Adam Csakai 2,5, Shuangshuang Jiang 1,5 ${ }^{1,}$ Christina Smith², Hiromi Tanji ${ }^{3}$, Jian Huang ${ }^{1}$, Torey Jones ${ }^{2}$, Kentaro Sakaniwa ${ }^{3}$, Lindsey Broadwell ${ }^{2}$, Chengrui Shi', Subada Soti ${ }^{2}$, Umeharu Ohto (i) ${ }^{3}$, Yaohui Fang ${ }^{4}$, Shu Shen (i) ${ }^{4}$, Fei Deng (D) ${ }^{4}$, Toshiyuki Shimizu (i] ${ }^{3}$ \& Hang Yin (iD) $1,2 \otimes$

Small-molecule modulators of TLR8 have drawn much interests as it plays pivotal roles in the innate immune response to single-stranded RNAs (ssRNAs) derived from viruses. However, their clinical uses are limited because they can invoke an uncontrolled, global inflammatory response. The efforts described herein culminate in the fortuitous discovery of a tetrasubstituted imidazole CU-CPD107 which inhibits R848-induced TLR8 signaling. In stark contrast, CU-CPD107 shows unexpected synergistic agonist activities in the presence of SsRNA, while CU-CPD107 alone is unable to influence TLR8 signaling. CU-CPD107's unique, dichotomous behavior sheds light on a way to approach TLR agonists. CU-CPD107 offers the opportunity to avoid the undesired, global inflammation side effects that have rendered imidazoquinolines clinically irrelevant, providing an insight for the development of antiviral drugs.

\footnotetext{
${ }^{1}$ School of Pharmaceutical Sciences, Key Laboratory of Bioorganic Phosphorous Chemistry and Chemical Biology (Ministry of Education), Department of Chemistry, Beijing Advanced Innovation Center for Structural Biology, Tsinghua-Peking Joint Center for Life Sciences, Tsinghua University, Beijing, China. ${ }^{2}$ Department of Chemistry \& Biochemistry and the BioFrontiers Institute, University of Colorado Boulder, Boulder, CO, USA. ${ }^{3}$ Graduate School of Pharmaceutical Sciences, The University of Tokyo, Tokyo, Japan. ${ }^{4}$ Wuhan Institute of Virology, Chinese Academy of Sciences, Wuhan, China. ${ }^{5}$ These authors

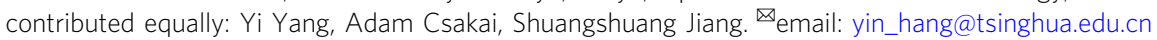


T oll-like receptors (TLRs) are highly conserved and crucial components of the innate immune system ${ }^{1,2}$. TLR3, 7, 8, and 9 located on endosomal membranes can recognize nucleic acids from viruses or bacteria, triggering potent antiviral and antitumor immune responses. Particularly, human TLR8 mediates antiviral immunity by recognizing ssRNA viruses ${ }^{3-5}$. While TLR1, 2, 4, 5, and 6 expressed on the plasma membrane can recognize extracellular stimuli. Once detected, these signals trigger an inflammatory response through transcription factors, such as NF- $\mathrm{kB}$ and interferon regulatory factors (IRFs). Activation of these transcription factors results in the production of pro-inflammatory cytokines and type I interferons, which are crucial in the activation of adaptive immunity ${ }^{6}$. The immune response is paramount to the host organism's ability to defend itself against invading pathogens, and underactivation of TLRs leaves the host vulnerable to widespread infection ${ }^{7}$. However, this immune response can be a double-edged sword. Aberrant activation of TLRs results in an overabundance of inflammatory factors that may lead to several autoimmune disorders and septic shock or even killing the host ${ }^{8,9}$.

Recent work has shown that TLR8 can uniquely induce a robust inflammatory response in newborns, who are particularly vulnerable to infection ${ }^{10}$. TLR agonists are promising vaccine adjuvants and antiviral agents, as TLR activation can control the induction, extent, and duration of an adaptive immune response through the production of cytokines ${ }^{6}$. For example, monophosphoryl lipid A activates TLR4 which is one of the FDA-approved adjuvants $^{11}$. Despite the lack of formal approval, it has been observed that many vaccines contain TLR agonists which act as adjuvants, including the Influvac and yellow fever vaccines ${ }^{12-14}$. TLR8 is a particularly enticing target due to its expression on myeloid dendritic cells (mDCs) and the proven capacity to activate the Type 1 helper $\mathrm{T}$ cells (Th1) cell-mediated immune response ${ }^{6}$. Particularly, TLR8 also plays an important role in the antiviral immune response through recognition of viral RNA in endocytic compartments ${ }^{15}$.

Enhancing the body's innate immunity through TLR7 or TLR8 has been proved to be an effective antivirus strategy ${ }^{3,5,16}$. R848, a TLR7 and TLR8 dual agonist ${ }^{17}$, also known as Resiquimod, is an FDA-approved drug formulated as a topical gel for the treatment of skin lesions caused by the herpes simplex virus and cutaneous T-cell lymphoma. Naturally, these small molecules have been tested as potential vaccine adjuvants and antiviral reagents, but few are suited for pharmacological development ${ }^{6}$ because they can cause nonspecific, global inflammation, and poor immune recruitment adjacent to the injection. Despite these shortcomings, imidazoquinolines are a prudent starting point in the search for TLR8-selective modulators, especially with their strong affinity and known binding mode ${ }^{18}$. However, designing TLR8 selective modulators has been a challenge due to the high degree of homology with TLR7. While few specific TLR8 antagonists are present in the literature ${ }^{19-21}$, imidazoquinoline-based agonists have been widely used for the study of TLR8 and TLR $7{ }^{17}$. Specifically, these compounds bind to TLR8 via utilizing hydrophobic interactions at the 2-position R-group, and three key hydrogen bonds are facilitated by Asp 543* and Thr 574*. Pistacking also occurs between Phe 405 and the quinoline ring of the scaffold, respectively (Supplementary Fig. 1).

In this work, we showed a series of rationally designed TLR8specific small molecules with unique synergistic activities in the presence of ssRNA, but inactive without the aid of ssRNA, making them potential antiviral reagents with characteristics distinct from imidazoquinolines. By considering the structural differences between the homologous TLR7 and TLR8, we identified a small-molecule scaffold that is tunable in both potency and specificity. Finally, we further assayed the lead compound CU-CPD107 in both cellular and biophysical systems to ascertain its mechanism and function.

\section{Results}

Design and rationale. The original discovery of imidazoquinoline agonists included many selective TLR7 agonists ${ }^{22-26}$ and nonselective TLR7/8 agonists $3,22,23,26$, but few TLR8-selective agonists ${ }^{27}$. Consistent across these molecules is a fused 2aminopyridine moiety, and most of structure-activity relationship (SAR) studies involved modifications on the remaining fivemembered ring of the scaffold. From these studies, we can see that a selective TLR7 agonist R837 can be modified to gain TLR8 activity via the incorporation of a hydroxyl group and an ethoxymethyl chain, compared with R848 (Fig. 1). If TLR8 selectivity was to be obtained, we assumed it necessary to maintain these two additions. Since the original imidazoquinoline synthesis yielded little compounds lacking TLR7 activity, we thought it best to seek TLR8 selectivity via SAR studies of the little-explored 2aminoimdazole moiety which had already produced a TLR8specific agonist and a pan-TLR inhibitor ${ }^{27}$.

In our opinion, the most interesting approach for this SAR study is to break apart the fused ring system of the quinoline ring to give a tetrasubstituted imidazole (Fig. 1). This design may most probably maintain the potential for all the previously discovered imidazoquinoline binding interactions to occur but also allows for additional flexibility from the newly rotatable bonds at the 4-position and 5-position of the imidazole ring. A wide range of substitutions could be installed at the 4-position to provide a small library of compounds. We envisioned a variety of carboxylate derivatives as suitable bioisosteres for the 2aminoquinoline moiety, and also found interest in functional groups that were unlikely to reproduce 2 -aminoquinoline behavior, such as simple aliphatics and halogens. We believe that this strategy would open the possibility for the discovery of both agonists and antagonists since the proposed molecules could theoretically fit the same imidazoquinoline binding site, but potentially in a different configuration.

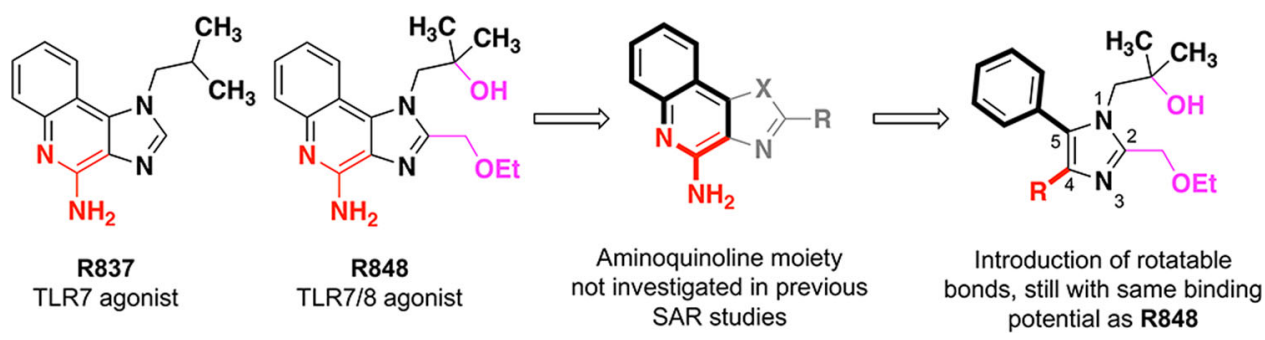

Fig. 1 Design and rationale of tetrasubstituted imidazole derivatives. The structure of R837 (a selective TLR7 agonist) and R848 (a nonselective TLR7 and TLR8 agonist) are shown. Previous SAR efforts resulted in R848 and R837 mainly focused on the 5-membered azole ring and its substituents. In this work, we broke apart the aminoquinoline ring to introduce more rotatable bonds but maintained the substituents that originally provided TLR 8 activity. 
Table 1 Analysis of the structure-activity relationship of the tetrasubstituted imidazole analogs.<smiles>[R]c1ccc(-c2c([R])nc(COCC)n2CC([R])(C)C)c([R])c1[R]</smiles>

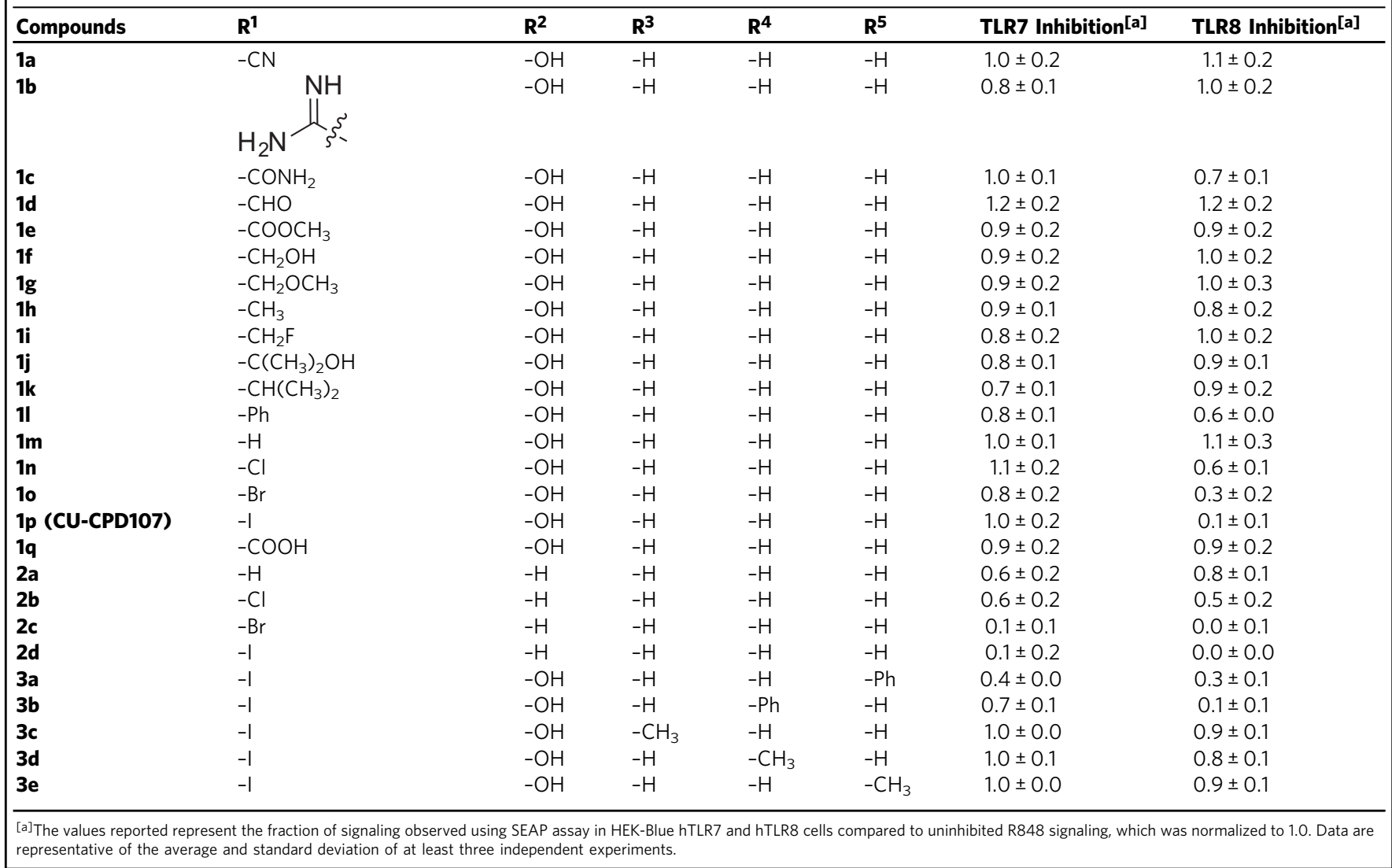

Synthesis of imidazoquinoline derivatives. The biggest challenge in the synthesis of a tetrasubstituted imidazole scaffold is the regioselective functionalization of positions $1,2,4$, and 5 . Given our previously described target design, it was also important for us to have 4-position functionalized with a group that would be inert to functionalization chemistry at the other imidazole positions, and could then be easily modified to give rise to several other 4-position functional groups. The latter approach was employed in a synthesis, all the compounds in Table 1 were synthesized according to Supplementary Information. Taking CU-CPD107 as an example (Fig. 2), it was started from the alkylation of imidazole with isobutylene oxide, as previously reported $^{28}$. The newly formed tertiary alcohol required a protecting group that would last for the duration of the remaining synthesis, only to be removed in the last step. For this task, we chose to convert the alcohol into the tert-butyldimethylsilyl (TBDMS) ether with TBDMS triflate and 2,6-lutidine. Next, we would take advantage of the acidity at the 2-position of the imidazole by $n$-butyl lithium, and quenching with $N, N$-dimethylformamide (DMF) to give a 2-formylimidazole. Without purification, we carried out aldehyde reduction, followed by Williamson ether synthesis which successfully added the ethoxymethyl chain at 2-position. Then, we took advantage of the superior nucleophilicity of the 4-position and treated the imidazole with $\mathrm{N}$-iodosuccinimide (NIS) to give the 4-iodo intermediate. With the neighboring iodine atom installed, a Suzuki coupling was carried out and finally removing protective groups with tetrabutylammonium fluoride (TBAF) in tetrahydrofuran (THF) to give CU-CPD107.

Engineering tunable selectivity and potency. With the rationale and synthetic routes above, we synthesized a series of tetrasubstituted imidazole derivatives (Table 1) and carried out SAR studies. With the first round of synthesis completed, all compounds were tested for their activity in HEK-Blue hTLR8 or hTLR7 cells activated by $100 \mu \mathrm{M}$ R848 using SEAP assay (Table 1). We did not observe agonism in either cell line. To our surprise, the carboxylate derivatives $(\mathbf{1 b}, \mathbf{1 c}, \mathbf{1 d}, \mathbf{1 e}$, and $\mathbf{1 q}$ in Table 1) and aliphatic analogs (1a, $\mathbf{1 f}, \mathbf{1 g}, \mathbf{1 h}, \mathbf{1 i}, \mathbf{1 j}, \mathbf{1 k}$, and $\mathbf{1} \mathbf{m}$ in Table 1) had little to no effect on R848-induced signaling at 100 $\mu \mathrm{M}$, despite having such structural similarities to the imidazoquinolines. However, there was a clear inhibitory trend observed within the halogenated analogs (1m, 1n,1o, and CU-CPD107 in Table 1), favoring the larger, more polarizable iodo analog (CU-CPD107). To our delight, using CU-CPD107 we observed significant inhibition of R848-induced signaling in HEK-Blue 

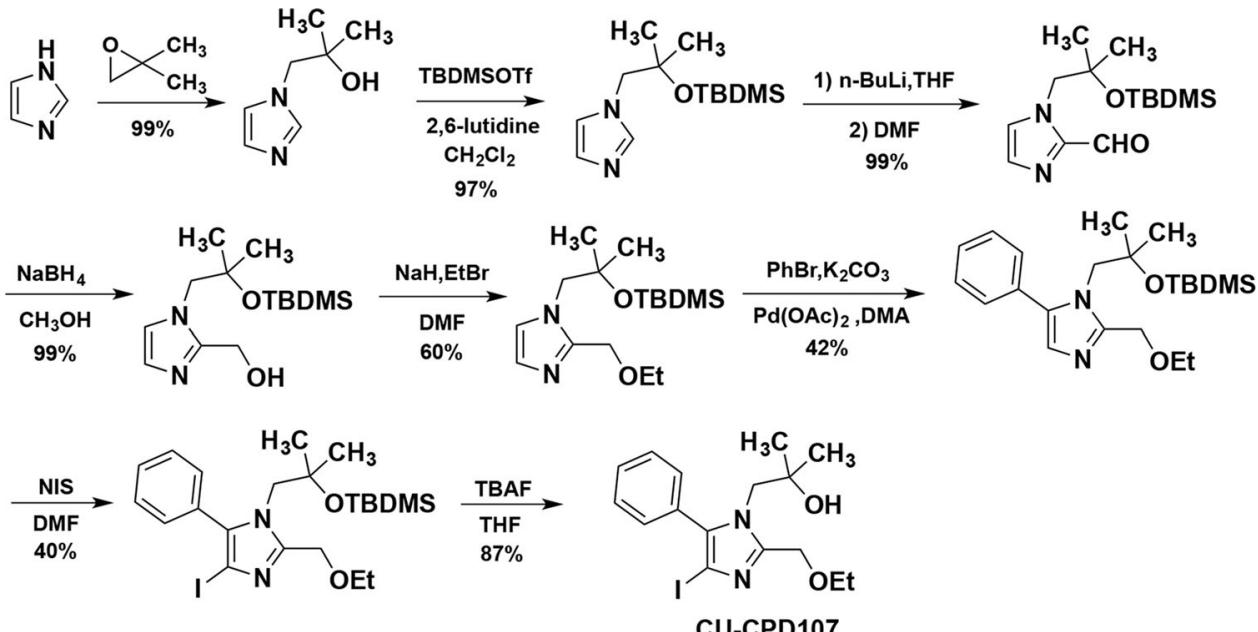

Fig. 2 Representative synthetic route of the imidazoquinoline derivatives, taking CU-CPD107 as an example. CU-CPD107 was synthesized in eight steps.

hTLR8 cells with an $\mathrm{IC}_{50}$ value of $13.7 \pm 1.1 \mu \mathrm{M}$ and no obvious molecules $(\mathbf{2 a}, \mathbf{2 b}, \mathbf{2 c}$, and $\mathbf{2 d}$ in Table 1 and Supplementary

a

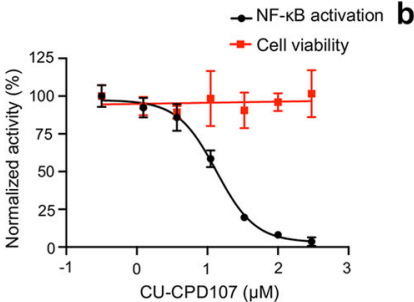

d

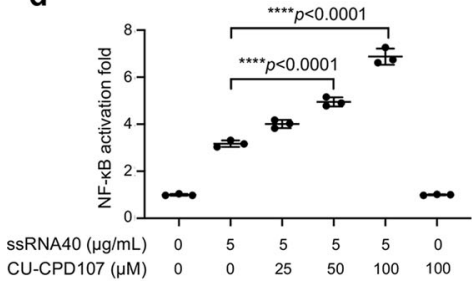

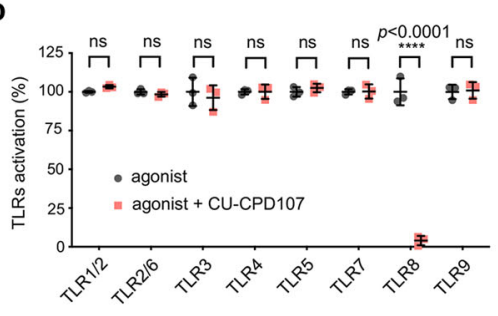

e

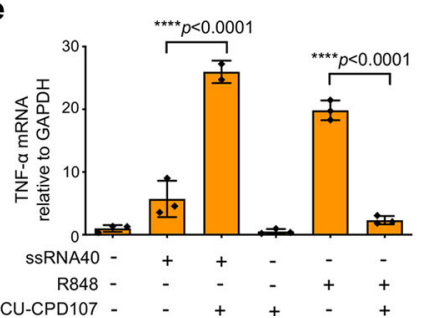

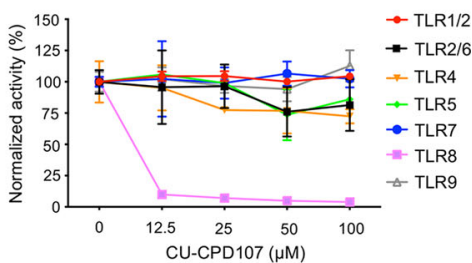

f

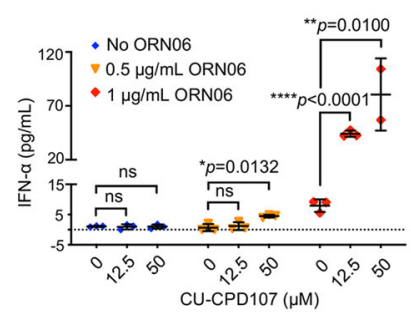

Fig. 3 Dichotomous behavior of CU-CPD107 in modulating the TLR8 signaling pathway. a The dose-response inhibition curve of $2.9 \mu \mathrm{M}$ R848-induced signaling in HEK-Blue hTLR8 cells is shown. Inhibition is clearly observed in HEK-Blue hTLR8 cells $\left(I C_{50}=13.7 \pm 1.1 \mu M\right)$ with no obvious toxicity up to 300 $\mu \mathrm{M}$. Data are mean \pm s.d.; $n=3$ independent experiments. $\mathbf{b}$ Specificity test for CU-CPD107 $(100 \mu \mathrm{M})$ using TLR-specific ligands to selectively activate the respective TLRs in HEK-Blue hTLRs cells for $24 \mathrm{~h}$. Data are mean \pm s.d.; $n=3$ independent experiments. c Specificity test of CU-CPD107 in PBMCs. Except for TLR8, CU-CPD107 alone did not inhibit other TLR pathways. Data are normalized to CU-CPD107 untreated cells. Data are mean \pm s.d.; $n=3$ independent experiments with independent blood donors. d CU-CPD107 could also synergistically activate TLR8 signaling in the presence of ssRNA40 $(5 \mu \mathrm{g} / \mathrm{mL})$ with a dose-dependent manner, while CU-CPD107 alone was unable to induce any activation. Signals are normalized to the untreated cells. Data are mean \pm s.d.; $n=3$ independent experiments. e CU-CPD107 could synergistically upregulate the mRNA levels of TNF- $\alpha$ in the presence of ssRNA40 $(5 \mu \mathrm{g} / \mathrm{mL})$, while it could inhibit R848-induced activation and itself had no effect in HEK-Blue hTLR8 cells. Data are mean \pm s.d.; the data shown are representative of three biologically independent experiments. $\mathbf{f}$ The synergistic effect of CU-CPD107 in human PBMCs stimulated with ORN06. The production of IFN- $\alpha$ was measured by ELISA. Data are mean \pm s.d.; the data shown are representative of three samples of independent blood donors examined over three biologically independent experiments. A one-way analysis of variance with Bonferroni's multiple comparisons test for multiple comparisons was used for statistical analysis. Statistical significance of the data is indicated as follows: ${ }^{\star} P<0.05,{ }^{\star \star} P<0.01,{ }^{\star \star \star} P<0.001,{ }^{\star \star \star \star} P<0.0001$; ns not significant. Source data of Fig. 3 are provided as a Source data file.

toxicity up to $300 \mu \mathrm{M}$ as detected by a WST1 assay (Fig. 3a) but had no effect on TLR7-mediated signaling. Intrigued by this newfound selectivity, we pursued more molecules bearing halogens at the 4-position of the imidazole. We thought an interesting hybrid R848/R837/CU-CPD107 molecule could be made by removing the hydroxyl group that is common to R848 and CUCPD107, but still maintaining the ethoxymethyl chain. Larger halogen substitutions at the 4-position decreased TLR7 and TLR8 signaling, however, this was due to increased toxicity of the
Table 1).

One challenge in developing modulators to target TLRs is to engineer specificity and potency. There are at least 13 homologous TLRs present in mice and 10 in humans ${ }^{29}$. We, therefore, tested CU-CPD107 against a panel of homologous hTLRs, including TLR1/TLR2, TLR2/TLR6, TLR3, TLR4, TLR5, TLR7, and TLR9 using TLR-specific ligands to selectively activate the particular TLR-overexpressing cells and human peripheral blood mononuclear cells (PBMCs). We found that CU-CPD107 
only inhibited synthesized small-molecule agonist-induced TLR8 signaling without affecting other TLRs, showing its high selectivity in intact cells (Fig. 3b, c).

CU-CPD107 unexpectedly converts to an agonist in the presence of ssRNA. Upon the completion of the second round of optimization, CU-CPD107 was identified as the most promising molecule among the entire series. While R848 is widely used to activate TLR7 and TLR8, it is not a natural ligand that has no biological relevance. So, it is important to test CU-CPD107 in the presence of ssRNA ligands which better emulate a viral infection. ORN06 and ssRNA40 are two commercially available GU-rich ssRNA known to be effective agonists of mouse TLR7 and human TLR8. Much to our surprise, we observed a remarkable reversal of activity. Rather than inhibiting ssRNA-induced TLR8 signaling which would be consistent with R848-induced signaling, we observed that CU-CPD107 behaved an agonist in the presence of ssRNA (Fig. 3d) in HEK-Blue hTLR8 cells, resulting in a fivefold activation at $100 \mu \mathrm{M}$ by SEAP assay relative to the control. Crucially, CU-CPD107 displayed no agonistic potential in the absence of ssRNA.

Next, we used a secondary cellular assay to confirm this unique, dichotomous behavior of CU-CPD107. TLR8 activation or inhibition can influence the downstream signaling transduction resulting in increased or decreased production of the cytokines $^{4}$. We investigated the effects of CU-CPD107 on the mRNA level of several downstream cytokines by reverse transcriptase quantitative PCR (RT-qPCR) in HEK-Blue hTLR8 cells. As shown in Fig. 3e and Supplementary Fig. 2, treatment with $100 \mu \mathrm{M}$ CU-CPD107 nearly abolished the elevation of IL-8, IFN- $\beta$, TNF- $\alpha$, IL- $1 \beta$, and IL- 6 mRNA levels induced by $22.8 \mu \mathrm{M}$ R848. By contrast, CU-CPD107 synergistically increased IFN- $\beta$, TNF- $\alpha$, IL-1 $\beta$, IL- 6 , and IL-8 mRNA expression levels in the presence of $5 \mu \mathrm{g} / \mathrm{ml}$ ssRNA40, whereas CU-CPD107 alone did not. It is important to note that ssRNA40 is a phosphorothioate (PS) modified RNA. To rule out the interference effects of PS, we further used ORN06 which is a native viral RNA sequence to confirm its synergistic activities with uridine as a positive control (Supplementary Fig. 3). The same behavior of CU-CPD107 was also observed in human PBMCs by co-treating cells with TL8-506 or ORN06 (Supplementary Fig. 4). These phenomena were further confirmed the dichotomous behavior of CU-CPD107 and exactly in agreement with the results in the SEAP assay.

Next, we detected the production of downstream cytokines in human PBMCs. To test the synergistic effect of CU-CPD107 in the presence of ssRNA in PBMCs, the production of IFN- $\alpha$ and TNF- $\alpha$ was measured by an enzyme-linked immunosorbent assay (ELISA). We found that the production of IFN- $\alpha$ was strongly and synergistically upregulated in PBMCs when co-treated with ORN06 and CU-CPD107 (Fig. 3f), which was in good agreement with the results shown in HEK-Blue hTLR8 cells. Besides, TNF- $\alpha$ could also be induced by CU-CPD107 in the presence of ORN06 (Supplementary Fig. 5). Furthermore, CU-CPD107 showed negligible cytotoxicity in PBMCs up to $100 \mu \mathrm{M}$ (Supplementary Fig. 6). To sum up, CU-CPD107, different from the conventional agonists, only activated TLR8-mediated signaling with the aid of ssRNA, thus may avoid invoking an uncontrolled, global inflammatory response.

Based on the promising preliminary results, we also attempted to explore the structure of CU-CPD107 to further understand its binding modes and structural constraints. We designed a series of analogs to extend the SAR studies around the benzene ring (3a, $\mathbf{3 b}, \mathbf{3 c}, \mathbf{3 d}$, and $\mathbf{3 e}$ in Table 1). Compounds $\mathbf{3 a}$ and $\mathbf{3 b}$ showed moderate inhibitory effects on both TLR7 and TLR8 signaling. Compounds $\mathbf{3 c}$, 3d, and $\mathbf{3 e}$ showed no inhibitory effects on both
TLR7 and TLR8 signaling but enhanced TLR8 signaling to more than fourfold with the aid of ssRNA compared to the control (Supplementary Fig. 7).

$\boldsymbol{K} \boldsymbol{d}$ determination. In order to investigate the binding affinity of CU-CPD107, a series of isothermal titration calorimetry (ITC) experiments were performed with the isolated ectodomain of human TLR8 (Supplementary Fig. 8). First, CU-CPD107 $(100 \mu \mathrm{M})$ alone was titrated into purified TLR8 $(10 \mu \mathrm{M})$, with no detectable change in heat observed (Supplementary Fig. 8a); R848 $(100 \mu \mathrm{M})$ alone was titrated into TLR8 $(10 \mu \mathrm{M})$ with an observed $K_{d}$ of $35.7 \mathrm{nM}$ (Supplementary Fig. 8b). For comparison, R848 $(100 \mu \mathrm{M})$ was titrated into TLR8 protein $(10 \mu \mathrm{M})$ premixed with CU-CPD107 $(100 \mu \mathrm{M})$, with no heat change observed (Supplementary Fig. 8c). These results indicated that CUCPD107 hindered R848 binding to TLR8, in agreement with the inhibition observed previously. Next, ORN06 $(200 \mu \mathrm{M})$ was titrated to TLR8 $\left(20 \mu \mathrm{M}\right.$ ) with a $K_{d}=0.9 \mu \mathrm{M}$ (Supplementary Fig. 8d). CU-CPD107 $(100 \mu \mathrm{M})$ was titrated into TLR8 protein $(10 \mu \mathrm{M})$ in the presence of ORN06 $(20 \mu \mathrm{M})$, showing an observed $K_{d}$ of $146 \mathrm{nM}$ (Supplementary Fig. 8e). Uridine $(1 \mathrm{mM})$ was also titrated to TLR8 $\left(20 \mu \mathrm{M}\right.$ ) with a $K_{d}=12 \mu \mathrm{M}$ (Supplementary Fig. 8f). While the $K_{d}$ value for uridine in the presence of the oligonucleotide was $2.7 \mu \mathrm{M}$ (Supplementary Fig. 8g) similar to the previous data ${ }^{30}$ which was about 18 -fold weaker than CU-CPD107. Finally, CU-CPD107 $(100 \mu \mathrm{M})$ was titrated to ORN06 $(40 \mu \mathrm{M})$ which did not show significant heat change (Supplementary Fig. 8h). Considering these results above, the heat change in Supplementary Fig. 8e would be derived from CU-CPD107 binding to TLR8, but not the binding to ssRNA. This suggested that ssRNA greatly enhanced CU-CPD107 binding to TLR8 in a similar manner as uridine did. Taken together, these results demonstrated that CU-CPD107 differentially affects R848 and ssRNA ligands binding to TLR8.

Dichotomous mechanism of CU-CPD107. Aiming to further understand the molecular mechanism of this unexpected, unique, and dichotomous behavior of CU-CPD107, we successfully obtained the crystal structure of the TLR8/CU-CPD107 complex (Fig. 4a). CU-CPD107 utilized hydrogen bonds with A518* and V520* (Fig. 4b, c). This newly identified compound CU-CPD107 inhibited TLR8 signaling via stabilizing its resting state which was the same mechanism with the first TLR8-specific inhibitor CUCPT8m we have reported previously ${ }^{19}$. The distances between the $\mathrm{C}$ termini of the two protomers of TLR8 dimer (TLR8/CUCPD107, $50 \AA$ ) was similar to that of the unliganded dimer (51 A). Superimposition of the TLR8 structure complexed with CU-CPD107 onto the corresponding unliganded TLR8 segment (residues 32-816) produced a root-mean-square deviation (RMSD) value of $1.2 \AA$.

Previous reports have shown a two-site activation mechanism for TLR8 with ssRNA degradation products ${ }^{8}$. The primary binding site recognizes dinucleotides (e.g., UG) which are found within the concave leucine-rich repeats of the TLR8 horseshoe structure. The secondary binding site is highly conserved, locating near the dimer interface which recognizes uridine. Even though uridine alone is inactive, its occupation of the secondary site is able to enhance TLR8 signaling in the presence of dinucleotides. Based on the observation that CU-CPD107 has the same behavior of that with uridine, we utilized the high-resolution X-ray crystal structure of human TLR8/ssRNA40 (PDB: 4R08) as a model and docked CU-CPD107 to the secondary binding site (Fig. 4d). The docking results suggested that CU-CPD107 interacts with G351 by additional hydrogen bonding and formed $\pi-\pi$ stacking with F405 (Fig. 4e, f). To validate the specific 
a Inactivated dimer (crystal structure)

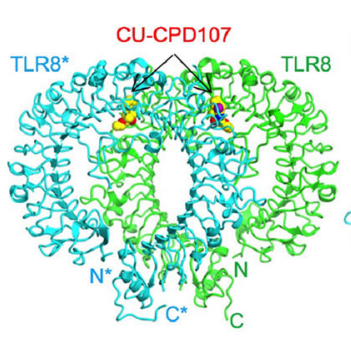

b

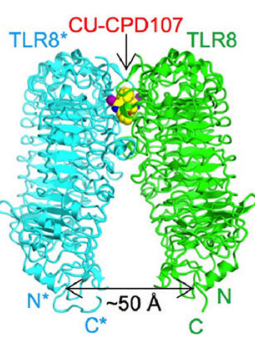

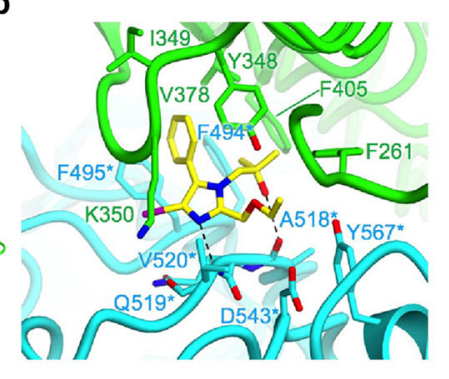

C

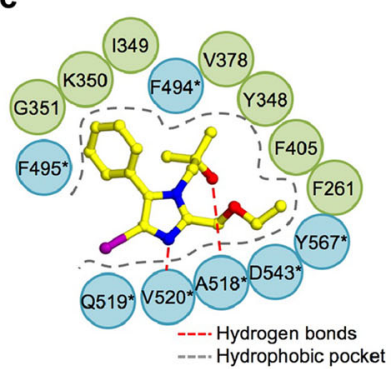

d

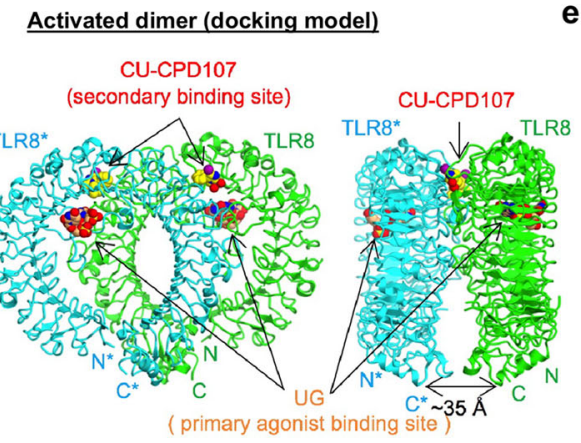

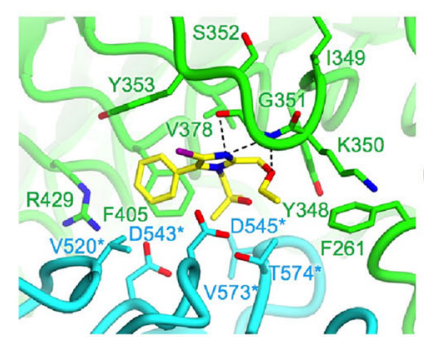

$\mathbf{f}$

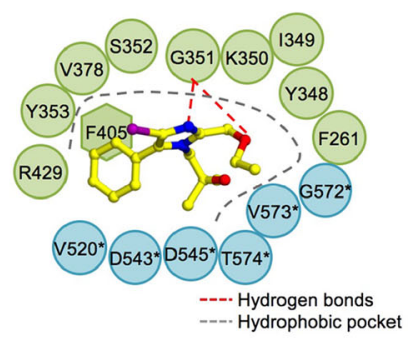

g

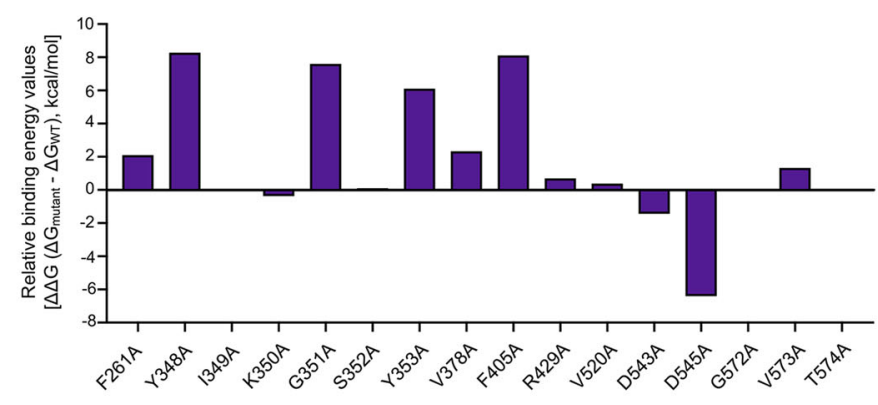

h

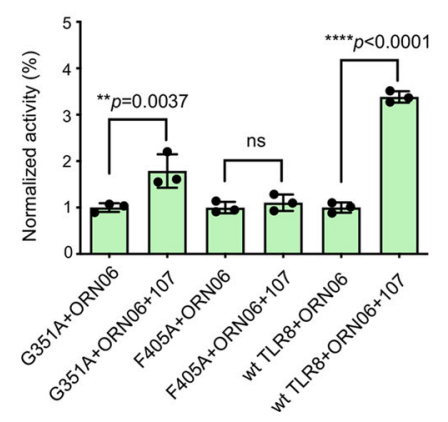

Fig. 4 Unexpected molecular mechanism of CU-CPD107. a Crystal structure of the TLR8/CU-CPD107 complex (PDB: 7CRF). Front (left) and side (right) views of the complex. TLR8 and its dimerization partner, TLR8 ${ }^{\star}$, are colored green and cyan, respectively. The ligand molecules are illustrated by spacefilling representations. The $\mathrm{C}, \mathrm{O}$, and $\mathrm{N}$ atoms of the ligands are colored yellow, red, and blue, respectively. $\mathbf{b}$ and $\mathbf{c}$ are the close-up views and schematic representation of interactions between CU-CPD107 and the TLR8 protein, respectively. The hydrophobic pocket and hydrogen bonds are shown as dashed gray arcs and dashed red lines, respectively. d Computational molecular modeling of CU-CPD107 docked to TLR8/ssRNA40 (PDB: 4R08) suggesting that the dimeric interface is the most likely target region for CU-CPD107. e and $\mathbf{f}$ are the close-up views and schematic representation of interactions between the CU-CPD107 with TLR8/ssRNA40 complex. $\mathbf{g}$ In silico alanine scanning of the residues within the CU-CPD107-binding site. The relative binding energy change $(\Delta \Delta G)$ of each mutant over the wild type was calculated using the Prime-MM/GBSA method. $\mathbf{h}$ Activities of CU-CPD107 in the mutants and wildtype hTLR8, stimulated by $5 \mu \mathrm{g} / \mathrm{mL}$ ORN06. The NF-KB induction fold of the mutants and wild-type TLR8 were analyzed by a SEAP assay using HEK-Blue Null1 cells. Data are mean \pm s.d.; $n=3$ independent experiments. A one-way analysis of variance with Bonferroni's multiple comparisons test for multiple comparisons was used for statistical analysis. Statistical significance of the data is indicated as follows: ${ }^{\star} P<0.05,{ }^{\star \star} P<0.01,{ }^{\star \star \star} P<0.001,{ }^{\star \star \star \star} P<0.0001$ ns not significant. Source data are provided as a Source data file.

interactions observed, we performed additional in silico alanine scanning of the residues within the CU-CPD107-binding site. The reduced association energy predicted with mutants harboring Y348A, G351A, Y353A, and F405A (Fig. 4g) agreed with the docking results and previous works ${ }^{18,31}$ that emphasizes the importance of the additional interactions in ligand recognition. Therefore, we rationalized that ssRNA converts TLR8 to its activated conformation, and CU-CPD107 binds to the secondary site instead of uridine. This binding by CU-CPD107 of the secondary site is much tighter than that of uridine, augmenting ssRNA-induced TLR8 activation. This ligand-induced signaling could be a beneficial adjuvant and antiviral agent, as the immune system would only be activated in the presence of ssRNA. To further confirm the function of the specific residues recognizing CU-CPD107, we experimentally mutated these residues and tested the activities of CU-CPD107 in HEK-Blue null1 cells transfected the mutant or wild-type plasmids using Lipofectamine 3000. When mutation of key residues to G351A and F405A, the synergistic activities were decreased (Fig. 4h), confirming that these residues were essential for binding to the CU-CPD107.

\section{Discussion}

The precise regulation of innate immunity plays a very important role in clinical application, especially in microbial infection which may induce a deadly inflammatory cytokine storm. The innate immune system is critical, not only for the first line of defense to fight against bacterial or viral infections but also for the activation of the adaptive immune response. Here, we reported the identification of a selective, dual-activity small-molecule CU-CPD107 
which demonstrated differential activity against the smallmolecule TLR8 agonists and ssRNA ligands. In the presence of R848, CU-CPD107 acted as a TLR8 signaling inhibitor. Not only did this inhibitor exhibit selectivity favoring TLR8 over the closely related TLR7 but it also showed no significant toxicity, which may be used as a chemical tool in cell-culture studies. Far more importantly, the activity of CU-CPD107 was reversed when TLR8 signaling was induced by ssRNA and synergistic agonism was observed. This was observed from both the SEAP assay at the cellular level and ITC assay at the protein level. In the absence of all other ligands, CU-CPD107 showed no pure agonistic activity, addressing a major challenge that has existed for previous TLR7 and TLR8 agonists as vaccine adjuvants or antiviral drugs. We believe this dichotomous characteristic should have great appeal beyond just vaccine adjuvants. This type of dormancy would be advantageous for any potential antiviral therapeutic, since only tissue affected with PAMPs, like ssRNA viruses, would elicit an inflammatory immune response and healthy tissue would remain unaffected, avoiding unwanted immune responses. This first-ofa-kind and previously unconsidered activity should change the way vaccine adjuvant and TLR researchers approach their own efforts in the future.

\begin{abstract}
Methods
Cell culture. HEK-Blue cells stably transfected with TLR1-TLR9 and an NF-kB SEAP reporter were used to assess the potency and specificity of compounds. HEKBlue hTLR cells were either purchased from Invivogen or prepared in-house. Cells were grown in Dulbecco's modified Eagle's medium (DMEM) media with $10 \%$ (v/v) heat-inactive FBS, $50 \mathrm{U} / \mathrm{ml}$ penicillin, and $50 \mathrm{mg} / \mathrm{mL}$ streptomycin. To select for TLR and reporter expression, HEK-Blue hTLR2 cells and HEK-Blue hTLR4 cells were maintained in presence of $100 \mu \mathrm{g} / \mathrm{mL}$ Normocin (Invivogen, No. ant-nr1) and $1 \times$ HEK-Blue Selection (Invivogen, No. hb-sel). Other HEK-Blue hTLR cells were grown in the complete media supplemented with $10 \mu \mathrm{g} / \mathrm{mL}$ blasticidin (Invivogen, No. ant-bl-05) and $100 \mu \mathrm{g} / \mathrm{mL}$ zeocin (Invivogen, No. ant-zn-05). HEK-Blue Null1 cells were grown in media in the presence of $100 \mu \mathrm{g} / \mathrm{mL}$ Normocin and $100 \mu \mathrm{g} / \mathrm{mL}$ Zeocin. All cells were incubated at $37^{\circ} \mathrm{C}$ in a humidified atmosphere of $5 \% \mathrm{CO}_{2}$
\end{abstract}

Secreted embryonic alkaline phosphatase (SEAP) assay. In total, 50,000 cells/ well were plated in a tissue-culture treated 96-well plate (Costar, No. 3596) in unsupplemented DMEM. Cells were then treated with $100 \mu \mathrm{M}$ R848 (InvivoGen, No. tlll-r848), $5 \mu \mathrm{g} / \mathrm{mL}$ ssRNA40/LyoVec (InvivoGen, No. tlrl-lrna40), and/or $5 \mu \mathrm{g} / \mathrm{mL}$ ORN06/LyoVec (InvivoGen, No. tlrl-orn6) and the indicated concentrations of the compounds. Cells were incubated overnight at $37^{\circ} \mathrm{C}$ in a humidified atmosphere of $5 \% \mathrm{CO}_{2}$ and assayed for NF- $\mathrm{kB}$ signaling. Cell media was assayed with Quanti-Blue (InvivoGen, No. rep-qbs3) per the manufacturer's recommendations. Specificity test in HEK-Blue hTLRs cells for CU-CPD107 using TLRspecific ligands to selectively activate the corresponding TLRs: $100 \mathrm{ng} / \mathrm{mL}$ of $\mathrm{Pam}_{3} \mathrm{CSK}_{4}, 100 \mathrm{ng} / \mathrm{mL}$ of $\mathrm{Pam}_{2} \mathrm{CSK}_{4}, 5 \mu \mathrm{g} / \mathrm{mL}$ of polyriboinosinic: polyribocytidylic acid [poly(I:C)], $20 \mathrm{ng} / \mathrm{mL}$ of LPS (lipopolysaccharide), $50 \mathrm{ng} / \mathrm{mL}$ of Flagellin, $2.9 \mu \mathrm{M}$ of R848, $22.8 \mu \mathrm{M}$ of R848, and $0.5 \mu \mathrm{M}$ of ODN2006 were used to selectively activate hTLR1/2, hTLR2/6, hTLR3, hTLR4, hTLR5, hTLR7, hTLR8, and hTLR9 cells, respectively. The SEAP reporter is constructed as a IFN- $\beta$ promoter fused to five NF- $\mathrm{kB}$ and AP-1-binding sites. SEAP was quantified by measuring absorbance at $620 \mathrm{~nm}$. Data were normalized with ligand + DMSO as $100 \%$ activity and untreated cells as 0 . Each data point represents the average and standard deviation of at least three biological replicates.

ELISA analysis in human PBMCs. Fresh whole blood donated from healthy volunteers was collected with informed consent under the Institution Review Board (IRB) of Peking Union Medical College Hospital (PUMCH)-approved protocol. PBMCs-related experiments have been described and approved by the IRB of PUMCH (No. S-478), consistent with Institutional Guidelines. Human PBMCs were separated from fresh blood using Density Gradient Centrifugation ${ }^{32}$. After separation, cells were seeded at a density of $6 \times 10^{5}$ cells/well in 96 -well plates immediately in $0.2 \mathrm{~mL}$ of Roswell Park Memorial Institute (RPMI) 1640 medium supplemented with $10 \%(\mathrm{v} / \mathrm{v})$ heat-inactive FBS, $100 \mathrm{U} / \mathrm{ml}$ penicillin, $100 \mu \mathrm{g} / \mathrm{L}$ streptomycin, $2 \mathrm{mM}$ L-glutamine, and $0.05 \mathrm{mM} 2$-mercaptoethanol and cultured at $37^{\circ} \mathrm{C}$ with humidified air containing $5 \% \mathrm{CO}_{2}$. Specificity test in PBMCs for $\mathrm{CU}$ CPD107 using TLR-specific ligands to selectively activate the corresponding TLRs: $10 \mu \mathrm{g} / \mathrm{mL}$ of $\mathrm{Pam}_{3} \mathrm{CSK}_{4}, 10 \mu \mathrm{g} / \mathrm{mL}$ of $\mathrm{Pam}_{2} \mathrm{CSK}_{4}, 0.5 \mathrm{mg} / \mathrm{mL}$ of LPS (lipopolysaccharide), $1 \mu \mathrm{g} / \mathrm{mL}$ of Flagellin, $0.5 \mathrm{mg} / \mathrm{mL}$ of R837, $1 \mu \mathrm{g} / \mathrm{mL}$ of TL8-506 and $5 \mu \mathrm{M}$ of ODN2006 were used to selectively activate hTLR1/2, hTLR2/6, hTLR4, hTLR5, hTLR7, hTLR8, and hTLR9 cells, respectively. Various concentrations of
CU-CPD107 $(0,12.5,25,50,100 \mu \mathrm{M})$ were added and co-incubated at $37^{\circ} \mathrm{C}$ in a humidified atmosphere of $5 \% \mathrm{CO}_{2}$. After $24 \mathrm{~h}$, the supernatant was collected and used for the measurement of TNF- $\alpha$ using BD OptEIATM human TNF-ELISA kit (BD Biosciences, No. 555212) according to the manufacturer's instructions. To test the synergism of CU-CPD107 in PBMCs stimulated by ssRNA, cells were incubated with indicated concentrations of ORN06 followed by the treatment of CUCPD107. The production of TNF- $\alpha$ ( $6 \mathrm{~h}$ after treatment) and IFN- $\alpha$ (24 h after treatment) in the supernatant were measured using BD OptEIATM human TNFELISA kit or human IFN- $\alpha$-ELISA kit (Multisciences, No. EK199-96) according to the manufacturers' instructions.

WST1 toxicity assay. Cellular toxicity was assessed using the Roche Cell Proliferation Reagent WST1 per the manufacturer's recommendation. Briefly, WST1 was diluted 1:10 into cell-containing media and assayed with absorbance at 450 $\mathrm{nm}$. Data were normalized with untreated cells as $100 \%$ viability, and 25\% DMSO treated samples as 0 . Each data point represents the average and standard deviation of at least three biological replicates.

RT-qPCR analysis. Seed HEK-Blue hTLR8 cells in a 12-well plate with $1 \times 10^{6}$ cells/well. Replaced the medium by fresh medium containing no serum after $24-\mathrm{h}$ incubation. Then, the cells were treated or untreated as below: ssRNA40 $(5 \mu \mathrm{g} / \mathrm{mL})$, ssRNA40 $(5 \mu \mathrm{g} / \mathrm{mL})$ with CU-CPD107 $(100 \mu \mathrm{M})$, CU-CPD107 $(100 \mu \mathrm{M})$ alone, R848 $(22.8 \mu \mathrm{M})$, or R848 $(22.8 \mu \mathrm{M})$ with CU-CPD107 $(100 \mu \mathrm{M})$, respectively. ORN06 $(5 \mu \mathrm{g} / \mathrm{mL})$ was also used in this experiment instead of ssRNA40, and uridine was used as a positive control. After 8-h treatment, cells were washed with PBS. TRIZOL reagent (Invitrogen, No. 15596026) was used to extract total RNA following standard protocols. iScript ${ }^{\mathrm{TM}} \mathrm{cDNA}$ Synthesis Kit (Bio-rad, No.1708890) and iTaq Universal SYBR Green Supermix (Bio-Rad, No.1725120) were used to perform reverse transcription and qPCR. TNF- $\alpha$, IFN- $\beta$, IL-1 $\beta$, IL-6, IL-8, and GAPDH primers were synthesized from Ruibiotech, and the primer sequences are listed in Supplementary Table 2 . The $\Delta \Delta \mathrm{Ct}$ method was used to analyze the data The relative mRNA levels of cytokine genes were compared with GAPDH gene and normalized to control. RT-qPCR performed in human PBMCs followed the similar procedure mentioned above. ORN06 $(2 \mu \mathrm{g} / \mathrm{mL})$ or TL8-506 $(1 \mu \mathrm{g} / \mathrm{mL})$ were used to stimulate the cells instead of ssRNA40 or R848. In all, $1 \times 10^{7}$ cells were collected to extract total RNA for each data point.

Protein expression, purification, and crystallization. The ectodomain of hTLR8 (residues 27-827) was prepared as described previously ${ }^{18}$. The sample was stocked in the concentration of $8.2 \mathrm{mg} / \mathrm{mL}$ in the solution of $150 \mathrm{mM} \mathrm{NaCl}$ and $10 \mathrm{mM}$ Tris- $\mathrm{HCl}(\mathrm{pH} 8.0)$. For the co-crystallization, we prepared the protein solution containing $7.0 \mathrm{mg} / \mathrm{mL}$ hTLR8 with an equimolar 12 mer phosphorothioate RNA (CCCCCGCCCCCC) and a tenfold excess of CU-CPD107 in the buffer composed of $150 \mathrm{mM} \mathrm{NaCl}, 10 \mathrm{mM}$ Tris- $\mathrm{HCl}(\mathrm{pH} 8.0)$, and 5\% DMSO. Crystallization trials were performed at $293 \mathrm{~K}$ with sitting-drop vapor-diffusion methods. Crystals of TLR8/CU-CPD107 were obtained by mixing the equivolume of the protein solution and the reservoir solutions containing $0.2 \mathrm{M} \mathrm{NaCl}, 20-27 \%$ (w/v) PEG 1000 , and $0.1 \mathrm{M}$ MES-NaOH (pH 6.8).

Data collection and structure determination. X-ray diffraction dataset of TLR8/ CU-CPD107 crystals was collected on the beamline PF NE3A at Photon Factory (Ibaraki, Japan). The experiment was conducted under the cryogenic condition at $100 \mathrm{~K}$, with a wavelength of $1.0000 \AA$. The dataset was processed using the XDS program ${ }^{33}$. The initial phase for TLR8/CU-CPD107 structure was determined by the molecular replacement method using the Molrep program ${ }^{34}$ with a search model based on the ligand-removed TLR8/CU-CPT8m structure (PDB ID: 5 WYX). Further model refinement was conducted with stepwise cycles of manual model building with the COOT program ${ }^{35}$ and restrained refinement with REFMAC $^{36}$ until the R factor was converged. CU-CPD107 molecules, N-glycans, and water molecules were built into the electron density map at the latter cycles of the refinement. The stereochemical quality of the final structure was evaluated with the PDB validation server (http://wwpdb-validation.wwpdb.org/). The Ramachandran plot showed that the favored and the allowed regions were $91 \%$ and $8 \%$ residues for TLR8/CU-CPD107, respectively. The electron-density map around CU-CPD107 is shown in Supplementary Fig. 9. The statistics of the data collection and refinement are summarized in Supplementary Table 3. The coordinate and the structure factor of TLR8/CU-CPD107 were deposited in the Protein Data Bank with PDB ID 7CRF.

Isothermal titration calorimetry (ITC) assay. ITC experiments were performed at $298 \mathrm{~K}$ in the buffer condition of $0.20 \mathrm{M} \mathrm{NaCl}, 25 \mathrm{mM}$ MES (pH 5.5), and $5 \%$ DMSO using a MicroCal iTC200 (GE Healthcare). The titration sequence started with a single injection $(0.4 \mu \mathrm{L})$ and was followed by 18 injections $(2 \mu \mathrm{L}$ each), spaced by $120 \mathrm{~s}$ between injections. The titration conditions were as follows: $100 \mu \mathrm{M}$ CU-CPD107 into $10 \mu \mathrm{M}$ hTLR8; $100 \mu \mathrm{M}$ R848 into $10 \mu \mathrm{M}$ hTLR8; $100 \mu \mathrm{M}$ R848 into $10 \mu \mathrm{M}$ hTLR8 in the presence of $100 \mu \mathrm{M}$ CU-CPD107; $100 \mu \mathrm{M}$ CUCPD107 into $10 \mu \mathrm{M}$ hTLR8 in the presence of $20 \mu \mathrm{M}$ ORN06; $1 \mathrm{mM}$ uridine into $20 \mu \mathrm{M}$ hTLR8; $200 \mu \mathrm{M}$ ORN06 into $20 \mu \mathrm{M}$ TLR8; $200 \mu \mathrm{M}$ uridine into $20 \mu \mathrm{M}$ TLR8 
and $40 \mu \mathrm{M}$ ORN06; $100 \mu \mathrm{M}$ CU-CPD107 into $40 \mu \mathrm{M}$ ORN06. The raw ITC data for each condition were processed by OrigineLab software (GE Healthcare).

Molecular docking. CU-CPD107 was docked against a complex of TLR8/ ssRNA40 (PDB: 4R08) by AutoDock 4.2 37 . Chem3D ultra 8.0 was used to construct the three-dimensional conformation structure of CU-CPD107. A box of $60 \times 60 \times$ 60-point grid (0.375- $\AA$ spacing between the grid points) and the affinity maps were generated and processed using AutoGrid 4.2 by default setting. For each docking case, 200 Lamarckian genetic algorithm runs were processed by default setting using AutoDock 4.2. The top-scored hit was chosen and visualized for further analysis. CueMol (http://www.cuemol.org) or PyMOL (http://www.pymol.org) was used to prepare all the molecular graphics.

In silico alanine scanning. The structure of the predicted TLR8/ssRNA40/CUCPD107 complex was processed by default setting using the Protein Preparation Wizard in Schrödinger suite 2018-138. The binding pocket was defined by identifying residues in direct contact with CU-CPD107, including F261, Y348, I349, K350, G351, S352, Y353, V378, F405, R429, V520, D543, D545, G572, V573, and T574. To validate the role of these residues in the ligand binding, each residue was mutated to alanine in silico within Maestro. Then, the relative binding free energy change $(\Delta \Delta G)$ of each mutant over the wild type was calculated using the Primemolecular mechanics/generalized Born surface area (MM/GBSA) method, keeping the ligand and other residues fixed.

Plasmids and mutagenesis. The hTLR8 expression plasmid (pcDNA3-TLR8) was constructed by inserting hTLR8 gene (from pcDNA3-TLR8-YFP) into the pcDNA3 expression vector. pcDNA3-TLR8-YFP was a gift from Doug Golenbock (Addgene plasmid \# 13024). Mutations were generated into pcDNA3-TLR8 plasmid via Fast Mutagenesis System kit (Transgen, No. FM111). All the sequences of hTLR8 and variants were confirmed by the sequence analysis at Genwiz Inc.

Activities of CU-CPD107 in mutant TLR8 and wtTLR8. HEK-Blue Null1 cells were seeded in 24 -well plates at a density of $1 \times 10^{4}$ cells per well. After $24 \mathrm{~h}$, media were replaced with fresh complete growth media and transiently transfected with pcDNA3-TLR8 or various mutant plasmids (500 ng) using Lipofectamine 3000 reagent (Invitrogen, No. L3000015). After $36 \mathrm{~h}$ transfection, cells were stimulated with $5 \mu \mathrm{g} / \mathrm{ml}$ ORN06, or $11.4 \mu \mathrm{M}$ R848 followed by the addition of $100 \mu \mathrm{M} \mathrm{CU}$ CPD107. After $24 \mathrm{~h}, 50 \mu \mathrm{L}$ of supernatant was collected in a new 96-well plate and add $50 \mu \mathrm{L}$ of Quanti-Blue. The mixture was incubated at $37^{\circ} \mathrm{C}$ for $20-40 \mathrm{~min}$, and the plate was measured on a Thermo Varioskan Flash microplate spectrophotometer at $620 \mathrm{~nm}$. Data were normalized as readouts of ligand-treated cells are $100 \%$ activation and untreated cells are 0.

Statistical analysis. Data are displayed as individual points or as mean \pm s.d., with sample size indicated in the figure legend. Statistical significance was evaluated using GraphPad Prism 6.0 for mac. A one-way analysis of variance with Bonferroni's multiple comparisons test for multiple comparisons or Student's two-sample unpaired $t$ test was used for statistical analysis. Statistical significance of the data is indicated as follows: ${ }^{*} P<0.05,{ }^{* *} P<0.01$, ${ }^{* * *} P<0.001$, ${ }^{* * * *} P<0.0001$; ns $=$ not significant.

Reporting summary. Further information on research design is available in the Nature Research Reporting Summary linked to this article.

\section{Data availability}

All data generated during this study are included in this manuscript and available for download. The final atomic coordinates and experimental structure factors were deposited in the Protein Data Bank with accession codes 7CRF for the TLR8/CUCPD107 complex. Structure information of TLR8/CU-CPT8m and TLR8/ssRNA40 mentioned in the manuscript are also available in the Protein Data Bank with accession codes $5 \mathrm{WYX}$ and 4R08, respectively. Source data are provided with this paper.

Received: 16 August 2020; Accepted: 21 June 2021;

Published online: 16 July 2021

\section{References}

1. O’Neill, L. A. \& Bowie, A. G. The family of five: TIR-domain-containing adaptors in Toll-like receptor signalling. Nat. Rev. Immunol. 7, 353-364 (2007).

2. Takeuchi, O. \& Akira, S. Pattern recognition receptors and inflammation. Cell 140, 805-820 (2010).

3. Jurk, M. et al. Human TLR7 or TLR8 independently confer responsiveness to the antiviral compound R-848. Nat. Immunol. 3, 499 (2002).
4. Heil, F. et al. Species-specific recognition of single-stranded RNA via toll-like receptor 7 and 8. Science 303, 1526-1529 (2004).

5. Triantafilou, K. et al. TLR8 and TLR7 are involved in the host's immune response to human parechovirus 1. Eur. J. Immunol. 35, 2416-2423 (2005).

6. Vasilakos, J. P. \& Tomai, M. A. The use of Toll-like receptor 7/8 agonists as vaccine adjuvants. Expert Rev. Vaccines 12, 809-819 (2013).

7. Hancock, R. E. W., Nijnik, A. \& Philpott, D. J. Modulating immunity as a therapy for bacterial infections. Nat. Rev. Microbiol. 10, 243-254 (2012).

8. Jimenez-Dalmaroni, M. J., Gerswhin, M. E. \& Adamopoulos, I. E. The critical role of toll-like receptors-from microbial recognition to autoimmunity: a comprehensive review. Autoimmun. Rev. 15, 1-8 (2016).

9. Mills, K. H. TLR-dependent T cell activation in autoimmunity. Nat. Rev Immunol. 11, 807-822 (2011).

10. Levy, O., Suter, E. E., Miller, R. L. \& Wessels, M. R. Unique efficacy of Toll-like receptor 8 agonists in activating human neonatal antigen-presenting cells. Blood 108, 1284-1290 (2006).

11. Ulrich, J. T. \& Myers, K. R. Monophosphoryl lipid A as an adjuvant. Past experiences and new directions. Pharm. Biotechnol. 6, 495-524 (1995).

12. Chua, B. Y. et al. Inactivated influenza vaccine that provides rapid, innateimmune-system-mediated protection and subsequent long-term adaptive immunity. mBio 6, e01024-01015 (2015).

13. Goff, P. H. et al. Synthetic Toll-like receptor 4 (TLR4) and TLR7 ligands as influenza virus vaccine adjuvants induce rapid, sustained, and broadly protective responses. J. Virol. 89, 3221-3235 (2015).

14. Querec, T. et al. Yellow fever vaccine YF-17D activates multiple dendritic cell subsets via TLR2, 7, 8, and 9 to stimulate polyvalent immunity. J. Exp. Med. 203, 413-424 (2006).

15. de Marcken, M., Dhaliwal, K., Danielsen, A. C., Gautron, A. S. \& DominguezVillar, M. TLR7 and TLR8 activate distinct pathways in monocytes during RNA virus infection. Sci. Signal 12, 2-18 (2019).

16. Wang, J. P. et al. Toll-like receptor-mediated activation of neutrophils by influenza A virus. Blood 112, 2028-2034 (2008).

17. Hemmi, H. et al. Small anti-viral compounds activate immune cells via the TLR7 MyD88-dependent signaling pathway. Nat. Immunol. 3, 196-200 (2002).

18. Tanji, H., Ohto, U., Shibata, T., Miyake, K. \& Shimizu, T. Structural reorganization of the Toll-like receptor 8 dimer induced by agonistic ligands. Science 339, 1426-1429 (2013).

19. Zhang, S. T. et al. Small-molecule inhibition of TLR8 through stabilization of its resting state. Nat. Chem. Biol. 14, 58-64 (2018).

20. $\mathrm{Hu}, \mathrm{Z}$. Y. et al. Small-molecule TLR8 antagonists via structure-based rational design. Cell Chem. Biol. 25, 1286 (2018).

21. Jiang, S. et al. Rationally designed small-molecule inhibitors targeting an unconventional pocket on the TLR8 protein-protein interface. J. Med. Chem. 63, 4117-4132 (2020).

22. Schiaffo, C. E. et al. Structure-activity relationship analysis of imidazoquinolines with Toll-like receptors 7 and 8 selectivity and enhanced cytokine induction. J. Med. Chem. 57, 339-347 (2014).

23. Larson, P. et al. Design and synthesis of N1-modified imidazoquinoline agonists for selective activation of Toll-like receptors 7 and 8. ACS Med. Chem. Lett. 8, 1148-1152 (2017).

24. Shukla, N. M. et al. 3-potent adjuvanticity of a pure TLR7-agonistic imidazoquinoline dendrimer. PLoS ONE 7, e43612 (2012).

25. Yoo, E. et al. 6-structure-activity relationships in Toll-like receptor 7 agonistic $1 \mathrm{H}$ imidazo[4,5-c]pyridines. Org. Biomol. Chem. 11, 6526-6545 (2013).

26. Beesu, M. et al. 8-structure-based design of human TLR8-specific agonists with augmented potency and adjuvanticity. J. Med. Chem. 58, 7833-7849 (2015).

27. Beesu, M. et al. Identification of a human Toll-like receptor (TLR) 8-specific agonist and a functional pan-TLR inhibitor in 2-aminoimidazoles. J. Med. Chem. 59, 3311-3330 (2016).

28. Torregrosa, R., Pastor, I. M. \& Yus, M. Solvent-free direct regioselective ring opening of epoxides with imidazoles. Tetrahedron 63, 469-473 (2007).

29. Rehli, M. Of mice and men: species variations of Toll-like receptor expression. Trends Immunol. 23, 375-378 (2002).

30. Alharbi, A. S. et al. Rational design of antisense oligonucleotides modulating the activity of TLR7/8 agonists. Nucleic Acids Res. 48, 7052-7065 (2020).

31. Tanji, H. et al. Toll-like receptor 8 senses degradation products of singlestranded RNA. Nat. Struct. Mol. Biol. 22, 109-115 (2015).

32. Ulmer, A. J., Scholz, W., Ernst, M., Brandt, E. \& Flad, H. D. Isolation and subfractionation of human peripheral-blood mononuclear-cells (Pbmc) by density gradient centrifugation on percoll. Immunobiology 166, 238-250 (1984).

33. Otwinowski, Z. \& Minor, W. Processing of X-ray diffraction data collected in oscillation mode. Methods Enzymol. 276, 307-326 (1997).

34. Vagin, A. \& Teplyakov, A. Molecular replacement with MOLREP. Acta Crystallogr. D. Biol. Crystallogr. 66, 22-25 (2010).

35. Emsley, P. \& Cowtan, K. Coot: model-building tools for molecular graphics. Acta Crystallogr. D. Biol. Crystallogr. 60, 2126-2132 (2004). 
36. Murshudov, G. N., Vagin, A. A. \& Dodson, E. J. Refinement of macromolecular structures by the maximum-likelihood method. Acta Crystallogr. D. Biol. Crystallogr. 53, 240-255 (1997).

37. Morris, G. M. et al. AutoDock4 and AutoDockTools4: automated docking with selective receptor flexibility. J. Comput. Chem. 30, 2785-2791 (2009).

38. Schrödinger, L. L. C., New York. Schrödinger Release 2018-1 (2018).

\section{Acknowledgements}

This work was funded by the National Natural Science Foundation of China Grand no. 21825702 (H.Y.), Tsinghua University Spring Breeze Fund Grant no 2020Z99CFY036 (H.Y.), Beijing Outstanding Young Scientist program Grand no. BJJWZYJH01201910003013 (H.Y.), International Research Project of Independent Research of Ministry of Education grand (UTokyo-Tsinghua Collaborative Research Fund) no. 2019Z02UTY (H.Y.) the Grant-in-Aid from the Japanese Ministry of Education, Culture, Sports, Science, and Technology Grant nos. 19 H00976 (T.S.), 18H04669, 19H03164, 19H04950 (U.O.), 19J21830 (K.S.), and by Core Research for Evolutional Science and Technology (T.S.). We thank the Beamline staff members at Photon Factory for their assistance with data collection. This research was partially supported by the Platform Project for Supporting Drug Discovery and Life Science Research (Basis for Supporting Innovative Drug Discovery and Life Science Research, BINDS) from AMED under grant number JP19am0101071 (support number 0030). We would also like to thank Dr. Shuting Zhang for producing of some HEK-Blue cells used in these studies.

\section{Author contributions}

H.Y. supervised the overall project. S.J., A.C., C.S., Y.Y., and J.H. designed the experiments in consultation with H.Y. S.J. and A.C. performed the chemical synthesis of the compounds. S.J., C.S., T.J., L.B., and S.S. performed the SEAP reporter assay and selectivity assay. Y.Y., J.H., and S.J. performed the ELISA, RT-qPCR, and the cell viability assay. H.T., K.S., U.O., and T.S. expressed the TLR8 protein, solved the crystal structure, and performed isothermal titration calorimetry experiments. J.H. performed the molecular docking. Y.Y. constructed plasmids of mutant and wild-type TLR8. C. Shi, Y.F., S. Shen, and F.D. are partially responsible for the cellular experiments. H.Y., S.J., A.C., C.S., Y.Y., and J.H. wrote the paper.

\section{Competing interests}

H.Y. and S.J. have filed a patent application based on the technology reported in this manuscript. The remaining authors declare no competing interests.

\section{Additional information}

Supplementary information The online version contains supplementary material available at https://doi.org/10.1038/s41467-021-24536-4.

Correspondence and requests for materials should be addressed to H.Y.

Peer review information Nature Communications thanks Gerhard Wolber, Michael Gantier and the other anonymous reviewer(s) for their contribution to the peer review of this work.

Reprints and permission information is available at http://www.nature.com/reprints

Publisher's note Springer Nature remains neutral with regard to jurisdictional claims in published maps and institutional affiliations.

(c) (i) Open Access This article is licensed under a Creative Commons Attribution 4.0 International License, which permits use, sharing, adaptation, distribution and reproduction in any medium or format, as long as you give appropriate credit to the original author(s) and the source, provide a link to the Creative Commons license, and indicate if changes were made. The images or other third party material in this article are included in the article's Creative Commons license, unless indicated otherwise in a credit line to the material. If material is not included in the article's Creative Commons license and your intended use is not permitted by statutory regulation or exceeds the permitted use, you will need to obtain permission directly from the copyright holder. To view a copy of this license, visit http://creativecommons.org/ licenses/by/4.0/.

(C) The Author(s) 2021 